\title{
BIOLOGICAL EVALUATION OF MUFFINS FORTIFIED WITH MICROENCAPSULATED IRON SALTS
}

(Received: 1. 8. 2012)

\author{
By \\ D. M. El-Nahal \\ Special Food and Nutrition Department, Food Technology Research Institute, \\ Agricultural Research Center, Giza, Egypt
}

\begin{abstract}
To avoid the development of color and flavor changes, rancidity and any unacceptable change in food fortified with iron salts. microencapsulated iron salts were used to protect the fortified food from these dislike characteristics. Therefore, the iron salts (ferrous fumarate, Fe Na EDTA, ferrous glouconate, ferrous citrate, ferrous carbonates and anhydrous ferrous sulphate) were microencapsulated with palm stearin, lecithin and ascorbic acid. For testing the efficiency of iron salts microencapsules, wheat flour (72 $\%$ extraction) was fortified with different microencapsules iron salts and the wheat flour fortified samples used to produce muffins. The chemical composition, Texture Profile Analysis (TPA), weight loss, sensory and biological evaluation of muffins prepared by wheat flour fortified with different microencapsules iron salts were estimated. The results indicated that there was no effect for wheat flour fortified with different microencapsules of iron salts on chemical composition, Texture Profile Analysis (TPA), weight loss, sensory evaluation of prepared muffins except the iron content compared to muffins prepared by unfortified wheat flour (MWF). It was increased in the fortified muffins. Depletion and repletion of iron biological experiments were designed. All male albino rats were fed on Fe deficient basal diet for six weeks. After the depletion period, the rats were divided randomly into 7 groups. Group 1: Fed on iron deficient basal diet and group 2: Fed on muffins prepared by unfortified wheat flour (MWF) and five groups fed on muffins prepared by wheat flour fortified with different microencapsules of iron salts for 30 days. Growth of rats, apparent digestibility coefficient (ADC), relative organs weight ratio, serum lipid profile, hematological evaluation and Fe absorption were measured. There was a significant increase in body weight, ADC, relative liver, kidney and heart weights ratio, hematological parameters, serum iron, total iron-binding capacity (TIBC), Fe kidney and Fe spleen of rats fed on muffins prepared by wheat flour fortified with different iron salts microencapsules compared to rats fed on Fe deficient basal diet and MWF. No significant difference was found in serum lipid profile among all tested rat groups. These results indicated that microencapsulation of iron salts had a positive effect to treat the dislike characteristics produced from fortified products with iron and had no effect on Fe absorbed and bioavailability.
\end{abstract}

Key words: bioavailability- iron absorbed-iron salts- microencapsulation- muffins.

\section{INTRODUCTION}

Anemia is considered as one of the most common index of nutritional deficiency worldwide and is caused by iron deficiency store or iron deficiency erythroanpoiesis. Several authors have reported that iron deficiency anemia (IDA) is mainly caused by some food constituents that may contribute to inhibition of iron absorption, hence contribute to the high prevalence of IDA (Lin et al., 2003). Iron is the most difficult mineral to add to foods and ensure adequate absorption.

The main problem is that the water soluble iron compounds (i.e. ferrous sulfate, ferrous lactate and ferrous gluconate), which are the most bioavailable, often lead to the development of unacceptable color and flavor changes in the food vehicle. When water-soluble compounds are added to cereal flours, for example, they often cause rancidity, and in low-grade salt, they rapidly lead to color formation. Insoluble compounds, 
such as elemental iron powders, on the other hand, do not cause sensory changes but may be so poorly absorbed as to be of little or no nutritional benefit.

Moreover, palm stearin is the solid fraction of palm oil that is produced by partial crystallization at controlled temperature (Lim, 2010). It is more variable in composition than palm olein, the liquid fraction of palm oil, especially in terms of its solid fat content, and therefore has more variable physical characteristics. Like crude palm fruit oil, palm stearin contains carotenoids, but physically refined palm oils do not, as they are removed or destroyed in the refining process. It is a useful source of natural hard vegetable fat for food applications. Palm stearin consists of mostly glyceryltripalmitate, with most of the rest of the fat content being glyceryl dipalmitate monooleate, (Kuo and Gardner, 2002). In terms of fatty acid composition, a typical soft palm stearin might contain almost $50 \%$ palmitic acid and $35 \%$ oleic acid.

Microencapsulation is a process where thin films or polymer coats are applied to small solid particles or droplets, of liquids. The food industry employs microencapsulation to protect sensitive food components, prevent nutritional loss, preserve flavors and aromas and mask undesirable flavors. Microencapsules can be designed to release core material by mechanical fracture, temperature changes $\mathrm{pH}$ manipulation or a combination of such factors. Stability of microencapsules can be optimized through choice of coating material, encapsulation technique and amount of core. Materials used to encapsulate water soluble compounds include hardened fats and wax. In successful fortification programme, both the fortificant and the vehicle should be acceptable to the target population (BovellBenjamin and Guinard, 2003). For example, the sprinkle-sized particles of ferrous fumarate are coated with a mono or diglyceride (hydrogenated soy lipid) and this thin coating protects the iron from the food (and food from the iron) and also masks the taste of the iron. The contents of the packet are sprinkled on the food that is served to the child. The iron will not react with the food altering its appearance or taste because it is encapsulated. The coating will dissolve in the stomach, releasing the iron salt, to be absorbed along with iron contained in the foods that constituted the meal. The availability of the added iron for absorption will be affected by inhibitors and enhancers of iron absorption that might be present in a meal fed to infants and toddlers (Wegmuller et al.,2003). Knowledge of the bioavailability of iron supplements is therefore necessary for a sound approach to establishing strategies for meeting the needs of the absorbed iron in them.

Muffin is a sweet, high-calorie baked product which is highly appreciated by consumers due to its good taste and soft texture. Muffin batter is a complex fat-in-water emulsion composed of an egg, sucrose, water and fat mixture as the continuous phase and bubbles as the discontinuous phase in which flour particles are dispersed (Martinez-Cervera et al.,2012). Therefore, muffins are considered a suitable product to examine the success of fortified wheat flour with different microcapsulated of $\mathrm{Fe}$ sources and insurance about the quality of product for sensory evaluation, and bioavailability of iron.

2.1. Materials

- Refined, bleached and deodorized (RBD) palm stearin sample was obtained from Gulf Company for Industrial Oils Co., Suez, Egypt.

- Ferrous fumarate, $\mathrm{Fe} \mathrm{Na}$ EDTA, ferrous gluconate, ferrous carbonates, anhydrous ferrous sulphate and ascorbic acid were obtained from Sigma-Aldrich Company, St. Louis, USA.

- Lecithin was obtained from soybean pilot plant, Food Technology Research Institute, Agric. Res. Center, Giza, Egypt.

- Wheat flour (72\% extraction), sugar, egg, milk, sunflower oil, salt, bicarbonate of soda, vanillin were purchased from the local market, Dokki, Giza, Egypt.

- The analytical kits were obtained from Randox Laboratories Ltd., Diamond Road, Crumlin. Co., Antrim, United Kingdom, BT294QY.

\subsection{Methods}

\subsubsection{Production of microencapsules}

The coating material used for the encapsulation process consisted of palm stearin $(0.005 \%$ of Butylated Hydroxy Toluene, BHT added) with a melting point of $63{ }^{\circ} \mathrm{C}$. The lecithin $(1 \%)$ was added to the molten palm stearin together with the $\mathrm{Fe}$ to reduce the viscosity of microencapsules. The ratio between palm stearin: $\mathrm{Fe}$ substrate was of 3.5:1.5 (g/g), $50 \mathrm{ml}$ distilled water was added. Moreover, the ascorbic acid was added (1:1 molar ratio of $\mathrm{Fe}$ substance: ascorbic acid, Premkumar and Bowlus, 2003). The mixture was homogenized at $1250 \mathrm{rpm} / \mathrm{min}$ for $20 \mathrm{~min}$. Spray dryer granulator PGL-B was used to produce 
different $\mathrm{Fe}$ microencapsules. The microencapsules were stored at $-5{ }^{\circ} \mathrm{C}$ in polyethylene till use.

\subsubsection{Fortifying wheat flour with microencapsules of different iron salts}

The $\mathrm{Fe}$ microencapsules were added to the wheat flour after determination of the iron content by atomic absorption spectrophotometry (AAS) of each microencapsule to obtain $100 \mu \mathrm{g} \mathrm{Fe} / \mathrm{g}$ flour, (Yeung et al.,2005) and the fortified flour was used to prepare muffins.

\subsubsection{Manufacture of muffins}

Six muffin formulations were prepared. One was used as a control treatment (wheat flour $72 \%$ unfortified with $\mathrm{Fe}$ ) and the other five were prepared by replacing wheat flour with fortified flour with different microencapsulated $\mathrm{Fe}$. The quantities of the ingredients were: $100 \mathrm{~g}$ Wheat flour $72 \%, 27 \mathrm{~g}$ egg yolk, $54 \mathrm{~g}$ egg white, $50 \mathrm{~g}$ milk, $100 \mathrm{~g}$ sugar, $46 \mathrm{~g}$ sunflower oil, $4 \mathrm{~g}$ bicarbonate of soda, $3 \mathrm{~g}$ citric acid, $1.5 \mathrm{~g}$ salt and $0.5 \mathrm{~g}$ vanillin according to Martinez-Cervera et al.(2011). After baking, the muffins were left to cool at room temperature for $1 \mathrm{~h}$ on a rack in order to avoid moisture condensing on their undersurface.

\subsubsection{Gross chemical composition of muffins}

Protein, ether extract, ash, and crude fiber contents were determined (A.O.A.C., 2000). Total carbohydrates were calculated by difference.

\subsubsection{Texture Profile Analysis (TPA) and weight loss of muffins}

Crumb texture was determined by a universal testing machine (Cometech, type B, Taiwan). An aluminium $25 \mathrm{~mm}$ diameter cylindrical probe was used in a Texture Profile Analysis (TPA) double compression test to penetrate to $50 \%$ depth, at 1 $\mathrm{mm} / \mathrm{s}$ speed test. Cohesiveness, gumminess, chewiness, springiness and resilience were calculated according to Boume (2002).

The muffins were weighed before (W3) and after baking and cooling (W4) and the weight loss was calculated, Martinez-Cervera et al. (2012). Each formulation was prepared twice, on different days, and seven muffins from each batch (fourteen determinations) were measured.

\subsubsection{Sensory evaluation}

The samples of muffins were organoleptically evaluated according to Martinez-Cervera et al. (2012). Ten consumers rated the appearance, color, texture, sweetness, flavor and overall acceptance of each muffin sample.

\subsubsection{Bioassay of muffins}

Forty two male albino rats weighing $105 \pm 5 \mathrm{~g}$. were housed at Ophthalmology Research Institute, Giza, Egypt. All animals were kept under normal healthy conditions and fed on basal diet (Eder and Kirchgessner, 1996) for one week. Water and diet were provided ad libitum. Then, the animals were fed on $\mathrm{Fe}$ deficient basal diet with low $\mathrm{Fe}$ content $(5 \mathrm{mg} / \mathrm{Kg}$ diet, Diaz-Castro et al.,2011) for 6 weeks. After depletion period, the rats were reweighed and divided randomly into 7 groups each one consist of 6 rats and fed on as follows (for 30 days):

Group (1): Fed on iron deficient basal diet.

Group (2): Fed on muffin prepared by unfortified wheat flour (MWF).

Group (3): Fed on muffin prepared by wheat flour fortified with ferrous fumarate microencapsules (MWF+M ferrous fumarate) .

Group (4): Fed on muffin prepared by wheat flour fortified with $\mathrm{Fe} \mathrm{Na}$ EDTA microencapsules (MWF+M Fe Na EDTA).

Group (5): Fed on muffin prepared by wheat flour fortified with ferrous gluconate microencapsules (MWF+M ferrous gluconate).

Group (6): Fed on muffin prepared by wheat flour fortified with ferrous carbonates microencapsules (MWF+M ferrous carbonates).

Group (7): Fed on muffin prepared by wheat flour fortified with anhydrous ferrous sulphate microencapsules (MWF+M ferrous sulphate).

\subsection{Growth of rats}

The rats were weighed at zero time, after depletion period and during the experiment. The apparent digestibility coefficient (ADC) was calculated from the data on $\mathrm{Fe}$ intake and $\mathrm{Fe}$ fecal (on dry weight basis), according to the equation: $\mathrm{ADC}=[(\mathrm{Fe}$ intake $-\mathrm{Fe}$ fecal $) \times 100] / \mathrm{Fe}$ intake, (Alferez et al.,2006).

At the end of the experimental period, the rats were weighed and killed by diethyl ether. The carcasses were dissected. Liver, kidney, spleen, heart and lung were separated by careful dissection, washed using saline solution and weighed. The organs weight ratio was calculated as follows (Weight of organ / total body weight at the end of the experiment $\mathrm{x}$ 100).

\subsection{Biochemical assay}

Blood samples were collected from the animals. Heparin was used as an anticoagulant. The serum was separated after centrifugation for $10 \mathrm{~min}$ at $3000 \mathrm{rpm}$ and kept frozen at $-20^{\circ} \mathrm{C}$ for biochemical assays:

Serum triglycerides, total cholesterol, HDLcholesterol and LDL-cholesterol were determined using the methods described by Fossati and Prencipe (1982),Wastson (1960), Assmann (1979) and Wieland and Seidel (1983), respectively. VLDL-cholesterol was calculated according to 
Wallach (1992) using the following equation: VLDL - cholesterol $=$ serum triglycerides $/ 5$

\subsection{Hematological evaluation}

Blood samples were received in cleaned tubes contained ethylene diamine tetra acetic acid (EDTA) as anticoagulant. Leucocytes count $\left(\mathrm{WBC}_{\mathrm{s}}\right)$, red blood cells $\left(\mathrm{RBC}_{\mathrm{s}}\right)$, blood haemoglobin $(\mathrm{Hg})$ and haematocrite value (PVC) were estimated by Wintrobe (1967), Dacie and Lewis (1975), Leong et al.(2003) and Burch and Siegel (1971), respectively.

\subsection{Fe absorption}

Serum $\mathrm{Fe}$ concentrations and the total ironbinding capacity (TIBC) were determined according to the methods described by Feigin (1977) and the International Committee for Standardization in hematology (1978), respectively.

The rate of transferrin saturation was subsequently calculated using the following equation:

Transferrin saturation $(\%)=[$ Serum $\mathrm{Fe}$ concentration $(\mathrm{mg} / \mathrm{dl}) / \mathrm{TIBC}(\mathrm{mg} / \mathrm{dl})] \times 100$

The $\mathrm{Fe}$ content of rat organs and feces were determined by atomic absorption Spectrophotometer. The samples were previously mineralized by a wet ash method as described by Parker et al. (1967).

\subsubsection{Statistical analysis}

Each parameter was analyzed separately by using one-way analysis of variance (ANOVA). For determining differences between groups, the Duncan test was used. All $p$ values of $\leq 0.05$ were considered to be significant (Bouveresse et al., 2011).

\section{RESULTS}

According to the chemical analysis of muffins, the data in Table (1) clear that the percentages of protein, fat, crude fiber, ash, and total carbohydrate were similar approximately on dry weight basis. The muffins prepared by unfortified wheat flour (MWF) were considered the lowest content of iron. Moreover, there was a fairly difference in protein content. The Texture Profile Analysis (TPA) parameters were estimated and the results in Table (2) illustrate that there was a little difference in cohesiveness of all tested muffin samples. Moreover, wheat flour fortified with different microencapsules of Fe salts had no effect on muffin gumminess, chewiness, springiness and resilience. The values for weight loss were fairly different in tested muffin samples. The weight loss ranged from 13.75 to $16.48 \mathrm{~g}$. The data in Table (3) show that the sensory evaluation of different tested muffins had no significant effect on sensorial properties. It was observed that microencapsules of iron had a good effect to prevent changes of sensorial properties when the food was fortified with iron.

The data presented in Table (4) illustrate the initial weight, weight after depletion period, final weight and body weight gain. At the end of the experimental period, the final weight of tested rat groups increased. There was no significant difference in the final weight among rats fed on tested muffins prepared by wheat flour fortified with different encapsulated iron. There was a fairly increase in final body weight of rats fed on iron deficient basal diet or MWF. The Fe intake, $\mathrm{Fe}$ fecal and $\mathrm{Fe}$ absorbed were significantly different among tested rat groups. Fe intake and $\mathrm{Fe}$ fecal were the highest in rats fed on MWF+M ferrous gluconate. Moreover, the digestive utilization of iron was assessed by the apparent digestibility coefficient (ADC), as an index of the absorbed to ingested iron ratio. The results indicated that, there was no significant difference in ADC among rats fed on muffins prepared by wheat flour fortified with ferrous fumarate or $\mathrm{FeSO}_{4}$ microencapsules. There was a significant decrease in $\mathrm{ADC}$ in rats fed on $\mathrm{MWF}+\mathrm{M}$ ferrous gluconate or MWF+M ferrous carbonates. It could be noticed that the lowest ADC was observed in rats fed on basal diet or unfortified muffins.

The relative weight ratio of interior organs was affected with the iron absorption as shown in Table (5). There was no significant difference in relative weight ratio of kidney and heart in tested rat groups except rats fed on iron deficient basal diet or MWF. Relative weight ratio of liver and spleen had significant difference among tested rat groups fed on muffins prepared by wheat flour fortified with different microencapsules iron.

The data in Table (6) show that there was no significant difference in serum triglycerides, total cholesterol and HDL-cholesterol and VLDLcholesterol among all tested rat groups. The serum LDL-cholesterol had a little significant difference. These results indicated that palm stearin has no effect in lipid profile of the tested rats.

The data in Table (7) show the haematological parameters of the experimental rat groups. There was no significant difference in blood $\mathrm{WBC}_{\mathrm{s}}$, $\mathrm{RBC}_{\mathrm{s}}$, haemoglobin, PVC\% and PLT among rat groups fed on muffins prepared by wheat flour fortified with different encapsulated iron. There was a significant decrease in items of blood picture of rat groups fed on iron deficient basal diet or muffins without fortified iron. 
Table (1): Chemical composition of muffins prepared by wheat flour fortified with different microencapsules of iron salts and iron content on dry weight basis

\begin{tabular}{|c|c|c|c|c|c|c|c|}
\hline & Protein \% & Fat \% & $\begin{array}{c}\text { Crude } \\
\text { fiber } \%\end{array}$ & Ash\% & $\begin{array}{c}\text { Total } \\
\text { Carbohydrate\% }\end{array}$ & $\begin{array}{c}\text { Caloric value } \\
\text { (Kcal/100 g.) }\end{array}$ & $\begin{array}{c}\text { Fe content } \\
\text { (mg/100g.) }\end{array}$ \\
\hline 1 & 10.06 & 17.51 & 0.28 & 1.14 & 71.06 & 482.07 & 0.58 \\
\hline 2 & 10.32 & 17.25 & 0.24 & 1.38 & 70.81 & 478.77 & 6.33 \\
\hline 3 & 9.45 & 16.92 & 0.24 & 1.29 & 72.10 & 478.48 & 6.51 \\
\hline 4 & 9.81 & 17.47 & 0.23 & 1.31 & 71.18 & 481.19 & 6.56 \\
\hline 5 & 10.12 & 17.31 & 0.21 & 1.35 & 71.01 & 480.31 & 6.43 \\
\hline \multicolumn{7}{|c|}{10.099} \\
\hline
\end{tabular}

Table (2): Texture Profile Analysis (TPA) and weight loss of muffins prepared by wheat flour fortified with different microencapsules of iron salts

\begin{tabular}{|c|c|c|c|c|c|c|}
\hline & Cohesiveness & Gumminess & Chewiness & Springiness & Resilience & Weight loss (g.) \\
\hline 1 & 0.71 & 3.83 & 3.16 & 0.82 & 0.58 & 16.48 \\
\hline 2 & 0.68 & 4.17 & 3.34 & 0.74 & 0.51 & 14.59 \\
\hline 3 & 0.69 & 3.67 & 3.50 & 0.75 & 0.56 & 14.09 \\
\hline 4 & 0.56 & 3.54 & 3.14 & 0.69 & 0.58 & 13.75 \\
\hline 5 & 0.72 & 3.33 & 4.84 & 0.77 & 0.50 & 15.28 \\
\hline 6 & 0.69 & 4.08 & 3.13 & 0.77 & 0.52 & 15.11 \\
\hline
\end{tabular}

Table (3): Sensory evaluation of muffins prepared by wheat flour fortified with different microencapsules of iron salts

\begin{tabular}{|c|c|c|c|c|c|c|}
\hline & Appearance & Color & Texture & Flavor & Sweetness & General acceptance \\
\hline 1 & $9.20 \pm 0.40^{\mathrm{ab}}$ & $8.93 \pm 0.50^{a}$ & $9.00 \pm 0.40^{a}$ & $8.87 \pm 0.64^{a}$ & $8.73 \pm 0.23^{\mathrm{a}}$ & $44.73 \pm 1.33^{a}$ \\
\hline 2 & $8.73 \pm 0.11^{a b c}$ & $9.00 \pm 0.53^{a}$ & $8.47 \pm 0.31^{a}$ & $8.40 \pm 0.72^{a}$ & $8.67 \pm 0.41^{a}$ & $43.27 \pm 0.23^{a}$ \\
\hline 3 & $9.27 \pm 0.42^{a}$ & $8.80 \pm 0.20^{a}$ & $8.67 \pm 0.64^{a}$ & $8.87 \pm 0.42^{a}$ & $8.67 \pm 0.31^{a}$ & $44.27 \pm 0.14^{a}$ \\
\hline 4 & $8.53 \pm 0.11^{c}$ & $8.93 \pm 0.58^{a}$ & $8.93 \pm 0.31^{a}$ & $8.07 \pm 0.42^{a}$ & $8.73 \pm 0.31^{a}$ & $44.13 \pm 1.30^{a}$ \\
\hline 5 & $8.67 \pm 0.31^{b c}$ & $9.00 \pm 0.72^{a}$ & $8.67 \pm 0.31^{a}$ & $8.27 \pm 0.70^{a}$ & $8.67 \pm 0.31^{a}$ & $43.27 \pm 1.50^{a}$ \\
\hline 6 & $8.43 \pm 0.31^{c}$ & $8.67 \pm 0.64^{a}$ & $8.80 \pm 0.53^{a}$ & $8.60 \pm 0.35^{a}$ & $8.87 \pm 0.31^{a}$ & $43.53 \pm 1.92^{a}$ \\
\hline LSD & 0.5370 & 0.9851 & 0.7732 & 0.3548 & 0.6442 & 2.4565 \\
\hline
\end{tabular}

Any two means have the same letter in the same column did not significantly different at $P \leq 0.05$

3= MWF $+M$ Fe Na EDTA

4= MWF $+M$ ferrous gluconate

$2=\mathrm{MWF}+\mathrm{M}$ ferrous fumarate
$5=\mathrm{MWF}+\mathrm{M}$ ferrous carbonates

$6=\mathrm{MWF}+\mathrm{M}$ ferrous sulphate 


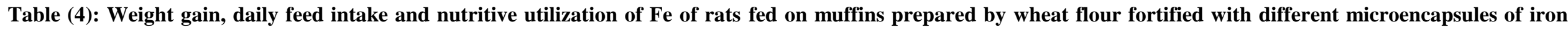
salts.

\begin{tabular}{|c|c|c|c|c|c|c|c|c|c|}
\hline Rat groups & $\begin{array}{c}\text { Initial } \\
\text { weight(g.) }\end{array}$ & $\begin{array}{c}\text { Weight after } \\
\text { depletion }\end{array}$ & $\begin{array}{c}\text { Final weight } \\
\text { (g.) }\end{array}$ & $\begin{array}{l}\text { Body weight } \\
\text { gain }(\mathrm{g})\end{array}$ & $\begin{array}{c}\text { Feed intake } \\
\text { (g./day) }\end{array}$ & $\begin{array}{c}\text { Fe intake } \\
(\mu \mathrm{g} / \text { day })\end{array}$ & $\begin{array}{c}\text { Fe fecal } \\
(\mu \mathrm{g} / \text { day })\end{array}$ & Fe absorbed* & ADC** \\
\hline $\mathbf{1}$ & $105.27 \pm 3.16^{\mathrm{a}}$ & $120.74 \pm 3.27^{\mathrm{a}}$ & $139.28 \pm 4.16^{b}$ & $18.54 \pm 1.01^{b}$ & $13.14 \pm 0.58^{b}$ & $68.35 \pm 3.02^{c}$ & $41.54 \pm 1.88^{\mathrm{e}}$ & $26.80 \pm 1.41^{\mathrm{c}}$ & $39.21 \pm 0.92^{c}$ \\
\hline 2 & $105.68 \pm 5.30^{\mathrm{a}}$ & $120.71 \pm 5.54^{\mathrm{a}}$ & $141.27 \pm 3.21^{b}$ & $20.23 \pm 1.90^{b}$ & $12.31 \pm 1.19^{b}$ & $71.44 \pm 6.94^{c}$ & $43.90 \pm 4.94^{\mathrm{e}}$ & $27.53 \pm 23.75^{c}$ & $38.54 \pm 3.07^{c}$ \\
\hline 3 & $105.21 \pm 2.51^{a}$ & $120.20 \pm 2.26^{\mathrm{a}}$ & $175.71 \pm 3.21^{\mathrm{a}}$ & $55.51 \pm 2.63^{a}$ & $15.94 \pm 0.32^{\mathrm{a}}$ & $1009.02 \pm 20.09^{a b}$ & $270.16 \pm 26.28^{\mathrm{cd}}$ & $738.86 \pm 26.85^{a b}$ & $73.23 \pm 2.50^{a}$ \\
\hline 4 & $105.15 \pm 5.73^{a}$ & $120.56 \pm 4.78^{a}$ & $181.00 \pm 3.25^{\mathrm{a}}$ & $60.44 \pm 6.31^{\mathrm{a}}$ & $15.31 \pm 1.49^{\mathrm{a}}$ & $996.02 \pm 95.500^{a b}$ & $297.79 \pm 20.45^{\text {bc }}$ & $698.89 \pm 82.46^{\mathrm{ab}}$ & $70.03 \pm 1.95^{a b}$ \\
\hline 5 & $106.10 \pm 3.81^{a}$ & $120.46 \pm 2.57^{\mathrm{a}}$ & $177.58 \pm 4.94^{\mathrm{a}}$ & $57.12 \pm 2.66^{a}$ & $16.65 \pm 1.32^{\mathrm{a}}$ & $1092.02 \pm 86.64^{a}$ & $377.37 \pm 24.04^{\mathrm{a}}$ & $714.65 \pm 103.32^{\text {ab }}$ & $65.23 \pm 4.36^{b}$ \\
\hline 6 & $105.30 \pm 4.34^{\mathrm{a}}$ & $120.10 \pm 2.59^{a}$ & $175.87 \pm 9.03^{\mathrm{a}}$ & $55.78 \pm 11.31^{\mathrm{a}}$ & $15.04 \pm 0.58^{a}$ & $967.29 \pm 37.36^{b}$ & $324.14 \pm 24.32^{b}$ & $643.15 \pm 60.95^{b}$ & $66.39 \pm 3.85^{b}$ \\
\hline 7 & $105.36 \pm 2.61^{a}$ & $121.53 \pm 2.99^{a}$ & $174.42 \pm 2.45^{\mathrm{a}}$ & $52.88 \pm 4.44^{a}$ & $16.04 \pm 0.67^{a}$ & $1023 \pm 42.75^{a b}$ & $258.10 \pm 14.85^{d}$ & $765.04 \pm 56.92^{a}$ & $74.71 \pm 2.48^{\mathrm{a}}$ \\
\hline $\mathrm{SD}(P \leq 0.05)$ & 25.288 & 6.336 & 8.678 & 9.734 & 1.508 & $\mathbf{8 8 . 7 7 7}$ & 35.611 & 99.336 & 5.139 \\
\hline
\end{tabular}

Any two means have the same letter in the same column did not significantly different at $P \leq 0.05$

$\begin{array}{ll}\text { 1- Iron deficient basal diet } & \text { 2=MWF (Muffin with unfortified wheat flour) }\end{array}$

$5=M W F+M$ ferrous gluconate

6= MWF $+M$ ferrous carbonates

$3=\mathrm{MWF}+\mathrm{M}$ ferrous fumarate

$7=\mathrm{MWF}+\mathrm{M}$ ferrous sulphate

4= MWF+M Fe Na EDTA

$*$ Fe absorbed $=$ Fe intake - Fe fecal

**ADC (apparent digestibility coefficient)

Table (5):Weights and relative ratio of rat organs fed on muffins prepared by wheat flour fortified with different microencapsules of iron salts.

\begin{tabular}{|c|c|c|c|c|c|c|c|c|}
\hline \multirow[t]{2}{*}{ Rat groups } & \multicolumn{2}{|c|}{ Liver } & \multicolumn{2}{|c|}{ Kidney } & \multicolumn{2}{|c|}{ Spleen } & \multicolumn{2}{|c|}{ Heart } \\
\hline & Weight (g.) & Relative ratio & Weight (g.) & Relative ratio & Weight (g.) & Relative ratio & Weight (g.) & Relative ratio \\
\hline 1 & $3.50 \pm 0.09^{c}$ & $0.023 \pm 0.002^{c}$ & $0.84 \pm 0.04^{b}$ & $0.006 \pm 0.0065^{b}$ & $0.376 \pm 0.04^{c}$ & $0.003 \pm 0.0029^{c}$ & $0.51 \pm 0.041^{b}$ & $0.004 \pm 0.0038^{b}$ \\
\hline 2 & $3.62 \pm 0.41^{c}$ & $0.026 \pm 0.003^{c}$ & $0.87 \pm 0.05^{b}$ & $0.006 \pm 0.0069^{b}$ & $0.400 \pm 0.03^{c}$ & $0.004 \pm 0.0030^{b c}$ & $0.53 \pm 0.041^{b}$ & $0.004 \pm 0.0039^{b}$ \\
\hline 4 & $6.79 \pm 0.64^{a}$ & $0.037 \pm 0.004^{\text {ab }}$ & $1.51 \pm 0.10^{\mathrm{a}}$ & $0.008 \pm 0.0087^{a}$ & $0.663 \pm 0.04^{\mathrm{a}}$ & $0.004 \pm 0.0039^{a}$ & $0.83 \pm 0.054^{\mathrm{a}}$ & $0.005 \pm 0.0049^{a}$ \\
\hline 5 & $5.97 \pm 0.06^{b}$ & $0.033 \pm 0.001^{b}$ & $1.42 \pm 0.11^{\mathrm{a}}$ & $0.007 \pm 0.0083^{a}$ & $0.563 \pm 0.08^{b}$ & $0.003 \pm 0.0036^{b}$ & $0.80 \pm 0.033^{a}$ & $0.005 \pm 0.0048^{a}$ \\
\hline 6 & $6.31 \pm 0.57^{\mathrm{ab}}$ & $0.035 \pm 0.003^{\mathrm{ab}}$ & $1.38 \pm 0.03^{\mathrm{a}}$ & $0.007 \pm 0.0083^{\mathrm{a}}$ & $0.664 \pm 0.01^{\mathrm{a}}$ & $0.004 \pm 0.0039^{a}$ & $0.80 \pm 0.081^{\mathrm{a}}$ & $0.005 \pm 0.0045^{\mathrm{a}}$ \\
\hline 7 & $6.70 \pm 0.17^{\mathrm{a}}$ & $0.038 \pm 0.001^{\mathrm{a}}$ & $1.49 \pm 0.10^{\mathrm{a}}$ & $0.008 \pm 0.0091^{\mathrm{a}}$ & $0.684 \pm 0.04^{a}$ & $0.004 \pm 0.0041^{\mathrm{a}}$ & $0.81 \pm 0.017^{\mathrm{a}}$ & $0.005 \pm 0.0047^{a}$ \\
\hline LSD $(P \leq 0.05)$ & 0.6686 & $\mathbf{0 . 0 0 4 3}$ & 0.1349 & 0.0008 & 0.0811 & 0.00004 & 0.0986 & 0.00004 \\
\hline
\end{tabular}

Any two means have the same letter in the same column did not significantly different at $P \leq 0.05$

1- Iron deficient basal diet 2=MWF (Muffin with unfortified wheat flour)

$6=M W F+M$ ferrous carbonates

$3=M W F+M$ ferrous fumarate

$7=M W F+M$ ferrous sulphate

4= MWF+M Fe Na EDTA 
Table (6): Lipid profile of rats fed on muffins prepared by wheat flour fortified with different microencapsules of iron salts

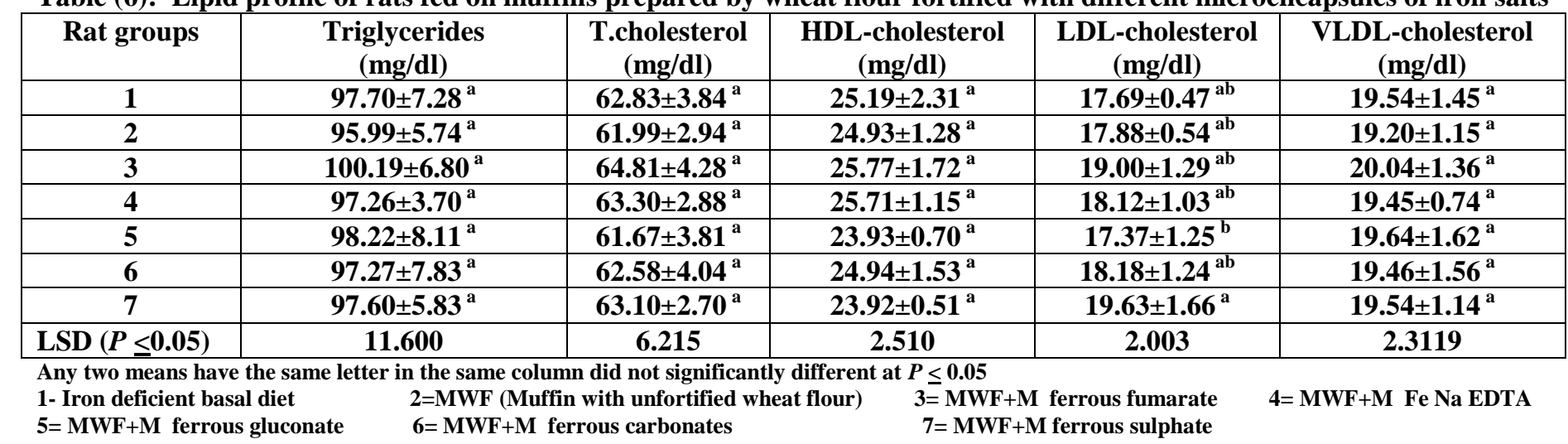

Table (7): Haematological parameters of rats fed on muffins prepared by wheat flour fortified with different microencapsules of iron salts

\begin{tabular}{|c|c|c|c|c|c|}
\hline Rat groups & WBC x10e $3 / \mathrm{ul}$ & $\mathrm{RBC}_{\mathrm{s}} \times 10 \mathrm{e}^{6} / \mathrm{ul}$ & Hemoglobin g/dl & PVC\% & PLT $\times 10 \mathrm{e}^{3} / \mathrm{ul}$ \\
\hline 1 & $6.37 \pm 0.60^{b}$ & $4.52 \pm 0.52^{b}$ & $7.22 \pm 0.31^{b}$ & $19.90 \pm 1.87^{b}$ & $143 \pm 3.61^{b}$ \\
\hline 2 & $6.86 \pm 0.85^{b}$ & $4.44 \pm 0.41^{b}$ & $7.43 \pm 0.59^{b}$ & $19.88 \pm 1.32^{b}$ & $149 \pm 5.86^{b}$ \\
\hline 3 & $11.96 \pm 0.59^{a}$ & $8.00 \pm 0.56^{a}$ & $13.88 \pm 1.19^{a}$ & $40.22 \pm 2.67^{a}$ & $396 \pm 28.88^{a}$ \\
\hline 4 & $11.97 \pm 0.79^{a}$ & $8.15 \pm 0.42^{a}$ & $12.60 \pm 0.96^{\mathrm{a}}$ & $38.08 \pm 4.03^{a}$ & $264 \pm 19.42^{\mathrm{a}}$ \\
\hline 5 & $11.62 \pm 0.78^{\mathrm{a}}$ & $7.61 \pm 0.57^{a}$ & $12.53 \pm 1.01^{\mathrm{a}}$ & $38.17 \pm 2.10^{a}$ & $393 \pm 15.18^{a}$ \\
\hline 6 & $11.40 \pm 0.61^{\mathrm{a}}$ & $7.93 \pm 0.44^{\mathrm{a}}$ & $12.70 \pm 0.89^{a}$ & $37.40 \pm 1.72^{\mathrm{a}}$ & $391 \pm 35.50^{\mathrm{a}}$ \\
\hline 7 & $12.38 \pm 0.57^{\mathrm{a}}$ & $7.63 \pm 0.84^{a}$ & $13.79 \pm 1.44^{\mathrm{a}}$ & $39.71 \pm 4.32^{a}$ & $404 \pm 9.45^{\mathrm{a}}$ \\
\hline LSD $(P \leq 0.05)$ & 1.217 & 0.973 & 1.693 & 4.893 & 37.372 \\
\hline
\end{tabular}

Any two means have the same letter in the same column did not significantly different at $P \leq 0.05$
$\begin{array}{ll}\text { 1- Iron deficient basal diet } \quad 2=M W F \\ \text { (Muffin with unfortified wheat flour) } \quad 3=M W F+M \text { ferrous fumarate }\end{array}$ ferrous gluconate $6=M W F+M$ ferrous carbonates $7=\mathrm{MWF}+\mathrm{M}$ ferrous sulphate

4= MWF+M Fe Na EDTA

$5=\mathbf{M W F}+\mathrm{M}$

Table (8): Iron absorption of muffins prepared by wheat flour fortified with different microencapsules of iron salts

\begin{tabular}{|c|c|c|c|c|c|c|c|c|}
\hline Rat groups & $\begin{array}{c}\text { Serum } \\
\operatorname{iron}(\mu / \mathrm{dl})\end{array}$ & TIBC $(\boldsymbol{\mu} / \mathrm{dl})$ & $\begin{array}{c}\text { Transferin } \\
\text { Sat. (\%) }\end{array}$ & $\begin{array}{c}\text { Fe liver } \\
(\mu \mathrm{g} / \mathrm{g})\end{array}$ & $\begin{array}{c}\text { Fe kidney } \\
(\mu \mathrm{g} / \mathrm{g})\end{array}$ & $\begin{array}{c}\text { Fe bone } \\
(\mu \mathrm{g} / \mathrm{g})\end{array}$ & $\begin{array}{c}\text { Fe heart } \\
(\mu \mathrm{g} / \mathrm{g})\end{array}$ & $\begin{array}{c}\text { Fe spleen } \\
(\mu \mathrm{g} / \mathrm{g})\end{array}$ \\
\hline 1 & $6.66 \pm 0.31^{b}$ & $21.33 \pm 0.75^{b}$ & $31.30 \pm 2.52^{c}$ & $80.99 \pm 3.98^{\mathrm{e}}$ & $40.49 \pm 2.34^{c}$ & $137.51 \pm 8.88^{d}$ & $40.54 \pm 3.47^{d}$ & $211.59 \pm 4.14^{\mathrm{c}}$ \\
\hline 2 & $6.34 \pm 0.74^{b}$ & $20.00 \pm 0.43^{b}$ & $31.64 \pm 3.08^{c}$ & $93.61 \pm 1.29^{d}$ & $58.08 \pm 3.61^{b}$ & $165.47 \pm 7.77^{c}$ & $60.04 \pm 2.46^{c}$ & $258.87 \pm 4.53^{b}$ \\
\hline 4 & $13.16 \pm 0.34^{a}$ & $26.34 \pm 0.63^{a}$ & $49.93 \pm 2.47^{\mathrm{ab}}$ & $168.43 \pm 2.90^{\mathrm{a}}$ & $86.57 \pm 2.74^{a}$ & $278.38 \pm 5.78^{a b}$ & $106.04 \pm 4.71^{b}$ & $461.53 \pm 8.75^{a}$ \\
\hline 5 & $12.06 \pm 1.07^{a}$ & $25.64 \pm 2.43^{\mathrm{a}}$ & $47.07 \pm 1.31^{b}$ & $161.29 \pm 3.07^{b}$ & $84.53 \pm 1.50^{a}$ & $271.00 \pm 6.41^{b}$ & $118.41 \pm 7.74^{a}$ & $460.63 \pm 6.46^{a}$ \\
\hline 6 & $12.23 \pm 0.50^{\mathrm{a}}$ & $26.37 \pm 0.85^{a}$ & $46.38 \pm 0.65^{b}$ & $155.66 \pm 2.35^{c}$ & $84.10 \pm 2.52^{a}$ & $265.37 \pm 5.80^{b}$ & $108.43 \pm 6.67^{\mathrm{ab}}$ & $463.23 \pm 2.28^{\mathrm{a}}$ \\
\hline 7 & $12.36 \pm 0.25^{\mathrm{a}}$ & $24.88 \pm 1.20^{\mathrm{a}}$ & $49.73 \pm 2.22^{\mathrm{ab}}$ & $169.09 \pm 4.25^{\mathrm{a}}$ & $88.87 \pm 3.07^{a}$ & $288.48 \pm 9.17^{\mathrm{a}}$ & $109.51 \pm 6.10^{\mathrm{ab}}$ & $463.30 \pm 6.16^{a}$ \\
\hline LSD $(P \leq 0.05)$ & 1.044 & 2.136 & 3.954 & 5.464 & 5.198 & 13.210 & 9.363 & 9.739 \\
\hline
\end{tabular}

Any two means have the same letter in the same column did not significantly different at $P \leq 0.05$

1 - Iron deficient basal diet

$2=$ MWF (Muffin with unfortified wheat flour)

$5=M W F+M$ ferrous gluconate

$6=\mathrm{MWF}+\mathrm{M}$ ferrous carbonates

$3=M W F+M$ ferrous fumarate

4= MWF $+M$ Fe Na EDTA 
Table (8) illustrates that rat groups fed on muffins prepared by wheat flour fortified with different iron microencapsules had no significant effect on serum iron, TIBC, Fe kidney and Fe spleen. There was a fairly significant difference in transferin saturation, Fe liver, $\mathrm{Fe}$ bone and $\mathrm{Fe}$ heart of the tested rat groups fed on muffins prepared from wheat flour fortified with different iron microencapsules. Generally, rats fed on iron deficient basal diet or MWF had significant lower values in all measured parameters. The results point out that MWF+M ferrous fumarate had the highest value of transferin saturation followed by $\mathrm{MWF}+\mathrm{M} \mathrm{Fe} \mathrm{Na}$ EDTA and MWF+M ferrous sulphate.

\section{DISCUSSION}

The obtained results indicated that gross chemical composition showed no difference among tested muffins except for the iron content. It was increased in muffins prepared from wheat flour with different microencapsules of iron salts compared with the MWF. Martinez-Cervera et al.(2011) cited that these ingredients of muffins were suitable to obtain muffins with good characters. Muffins are characterised by a typical porous structure and high volume which confer a spongy texture. To obtain such a final structure, a stable batter lodging many tiny air bubbles is required. The Texture Profile Analysis (TPA) parameters were not affected with fortified wheat flour with microencapsules of $\mathrm{Fe}$ sources. Chewiness is a secondary texture parameter that is associated with difficulty in chewing the sample and forming a bolus before swallowing (Baixauli et al.,2008). Moreover, Kiskini et al. (2012) showed that microencapsulated forms are not expected to bring about any negative effects on physical and sensory quality of gluten free bread. Sensory evaluation, one of the most important parameters to be taken into account in food fortification is the no change of the sensorial properties in fortified food. Several iron compounds with high bioavailability, in general, produce modifications on the taste and color of the fortified foods or they might produce rancidity because of fat oxidation (Yao et al.,2010). In this case, it could be noticed that the sensorial properties of the fortified muffins were not affected after the addition of the microencapsules of $\mathrm{Fe}$. The main target of microencapsulation process is to prevent any harmful effects on sensory parameters from the fortified materials (Diosady et al.,2002). Therefore, microencapsules of $\mathrm{Fe}$ had a good effect in sensory properties of fortified muffins.

Regarding the body weight gain and feed intake, it could be noticed that addition of $\mathrm{Fe}$ microencapsules in the muffins improved body weight and feed intake. There was a fairly increase in body weight of rats fed on iron deficient basal diet or MWF. Iron deficiency was accompanied with a decrease in weight gain and feed intake in rats. This result is in agreement with Strube et al.,2002, Hernandez et al.,2003 and Soliman et al.,2010. In contrast, the present data show that no significant difference was noticed in body weight gain of rats fed on different muffins prepared by wheat flour fortified with different microencapsules of iron. The supplement of muffins with iron improved the weight gain of anemic rats. These results are in agreement with those reported by Salgueiro et al.,2005 and Lobo et al.,2011). As described by Windisch (2002), the regulation of iron homeostasis occurs mainly at the digestive level, with hardly any renal excretion of this mineral taking place. Therefore, taking these facts into consideration, iron absorption would be a term synonymous with bioavailability. These results demonstrate that the muffins prepared by wheat flour fortified with microencapsules of ferrous fumarate, $\mathrm{FeSO}_{4}$ and $\mathrm{MWF}+\mathrm{M} \mathrm{Fe} \mathrm{Na}$ EDTA had the highest ADC. Iron compounds have different effects on $\mathrm{Fe}$ utilization. Hernandez et al. (2003) reported that iron compounds must be selected to fortify wheat flour when intended for public nutrition programs. Iron utilization was greater in rats fed on wheat flour supplemented with ferrous sulfate, followed by fumarate and citrate than in rats fed on reduced iron. Moreover, Hernandez et al.(2006) cited that iron bioavailability indicators were best in rats fed corn tortillas fortified with ferrous sulfate and $\mathrm{NaFe}$ (III)EDTA than in those fed unfortified corn tortillas.

The data in Table (5) illustrate that there was significantly higher relative weight ratios in liver, kidney and heart of rats fed on different muffins prepared by wheat flour fortified with different microencapsules of iron than rats fed on iron deficient basal diet or MWF. It was clear that iron maintained the normal weight of organs. Roodenburg et al.(1995) stated that weights of liver, spleen, heart and kidney were significantly higher in groups of rats fed on diets with iron, vitamin $\mathrm{A}$ and vitamin $\mathrm{C}$ fortification. Meanwhile, organ weights were lower in rat groups that received no dietary vitamin $\mathrm{A}$, vitamin $\mathrm{C}$ and any source of iron (Kelleher and Lonnerdal, 2005). 
Palm stearin (PS) is the solid fraction obtained by controlled temperature fractionation, and the liquid fraction is known as palm olein. PS can be used as a source of fully natural hard component in the manufacture of edible fat products, such as margarine and shortening. The data in Table (6) clearly indicate that there were no significant difference in parameters of serum lipid profile among all tested rat groups. This proves that palm stearin had no effect on the serum triglycerides, total cholesterol and HDL-cholesterol and VLDLcholesterol in the tested rats. It may be related to palm stearin that could be considered a negligible competent compared to the whole weight of muffin ingredient.

Formation of hemoglobin happens to be the chief function of iron. Not only that, being a part of hemoglobin, it gives the dark red shade to the blood and also aids in transporting oxygen to the body cells (Knutson and Wessling-Resnick 2003). All the haematological parameters studied, $\mathrm{WBC}_{\mathrm{s}}$, $\mathrm{RBC}_{\mathrm{s}}$, haemoglobin, PVC and PLT were within normal limits for the rats fed on muffins prepared by wheat flour fortified with different encapsulated iron at the end of the experimental period. After consuming iron deficient basal diet or muffins without fortified iron the rats were anaemic and the haematological parameters decreased under the normal limits. These results are in agreement with those reported by Alferez et al.,2006 and Lobo et al.,2011.

The source of iron is either from food supply (heme) or free iron from other sources. Kinds of iron are processed by the gut (stomach and intestine) where they are converted to a form of iron readily used by the body. Finally, the iron winds up in the intestinal epithelial cells, ready for export to red blood cells, muscle tissue and organs (Vulpe et al., 1999). The data in Table (8) clarify that fortification of wheat flour with the microcapsules of different iron sources had a positive effect to improve the absorbability of iron and treat the effect of depletion period of iron. Fe concentrations decreased in the serum and all the organs of anemic rats were analyzed, compared with tested rat groups fed on muffins prepared by wheat flour fortified with different encapsulated iron (diets supplied with iron). Similar findings were reported by other authors (Reeves et al.,2005; Alferez et al.,2006). Moreover, Urbano et al. (2007) cited that the Fe content in heart, kidney or femur did not maintain any correlation with the intestinal absorption of this mineral.

In summary, new microencapsulation techniques continuously emerge and the growing market for functional foods is the major driving force behind innovation in food technology field (Fang et al.,2005). Spray drying, freeze drying, air-suspension coating, and extrusion are considered methods to produce microcapsules (Gharsallaoui et al.,2007). Spray drying was chosen for the production of the microencapsules because of the desirable coating characteristics produced by the technique and because spray drying is currently the least expensive encapsulation technology (Gouin, 2004). Palm stearin was chosen as the coating material because of its high melting point $\left(63^{\circ} \mathrm{C}\right)$, which may allow the sprayed capsules to resist high temperatures encountered during manufacture, storage, transport and because of its hydrophobic properties, which prevent the entrance of water and reduce reactions between different core of microcapsule component, (Dary and Mora, 2002).

Several factors in the diet can influence the mineral bioavailability, the magnitude of which depends on inhibitors and promoters in a meal, and hence on the food matrix (Gibson, 2007). The presence of ascorbic acid in the microencapsules helps to increase absorbability and bioavailability of iron. Lee et al. (2003) showed that serum iron content and transferring saturation increased dramatically when subjects consumed milk containing both encapsulated iron and encapsulated ascorbic acid compared with those when consumed uncapsulated iron or encapsulated iron without ascorbic acid. Moreover, ascorbic acid is capable of reducing $\mathrm{Fe}$ (III) to Fe (II), (Wienk et al.,1999) and it is used as an absorption enhancer (Hurrell, 2002). Moreover, the source of iron had an effect on bioavailability of iron. All iron supplements are not the same. Ferrous iron is much better absorbed than ferric iron (Davidsson et al., 2000). The most common form of iron supplement is ferrous sulfate, but it is known to produce intestinal side effects such as constipation, nausea, and bloating in many users. Some forms of ferrous sulfate are enteric-coated to delay tablet dissolving and prevent some side effects, but enteric-coated iron may not be absorbed as well as iron from standard supplements. Ferrous iron is the form that is mostly used for correction of iron deficiency. About $3-5 \%$ of the iron present in alimentary canal in ferrous form is absorbed (Kurtoglu et al., 2003). Other forms of iron supplements, such as ferrous fumarate, ferrous gluconate, and iron glycine amino acid chelate (Fox et al., 1998) are readily absorbed and less likely to cause intestinal side effects. In order to overcome the problems 
(side effects) created from using tradition treatment of anemia, they tried to use iron chelated amino acids. Therefore, Salgueiro et al.(2005) illustrated that biological behavior of ferrous gluconate does not differ significantly from the reference standard (iron sulphate). Meanwhile, NaFeEDTA is a suitable fortificant for improving iron-deficiency status in developing countries (Igarashi et al.,2006). It is noteworthy the foods (infant formula, cereal, sugar and fish sauce) were consumed with an enhancer of iron absorption (EDTA) added to overcome absorption inhibitors (Hurrell, 2002), but the fortification occurred unacceptable change in foods.

Generally, microencapsules of iron have a fat coating which protects them from chemically reacting with unsaturated fats in the flour or meal, and be degraded by lipases in the gut so that the ferrous salt is available for absorption. The microencapsules of iron may have a large particle size causing them to be removed from flour during final sifting. But preliminary studies have shown them to be well absorbed, even with high extraction flour, and costs may drop if their use becomes more prevalent.

\section{REFERENCES}

Alferez M. J.M., Lopez-Aliaga I., Nestares T., Diaz-Castro J., Barrionuevo M., Ros P. B. and Campos M. S. (2006). Dietary goat milk improves iron bioavailability in rats with induced ferropenic anaemia in comparison with cow milk. International Dairy J. 16: 813-821.

A.O.A.C. (2000). Association of Official of Agricultural Chemists Official Methods of Analysis. 17 th. Ed., Pub. By the A.O.A.C., Washinton D.C.

Assmann G. (1979). A fully enzymatic colorimetric determination of HDLcholesterol in the serum. Internist 20:559.

Baixauli R., Sanz T., Salvador A. and Fiszman S.M. (2008). Muffin with resistant starch: Baking performance in relation to rheological properties of the batter. J. Cereal Sci. 47:502 - 509.

Boume T. (2002). Food texture and viscosity: Concept and measurement. 2 ed., Elsevier Science and Technology Books. P. 1-32.

Bouveresse D. J. R., Pinto R. C., Schmidtke L.M., Locquent N. and Rutledge D.N. (2011). Identification of significant factors by an extension of ANOVA-PCA based on multiblock analysis. Chemometrics and
Intelligent Laboratory Systems 106:173 182.

Bovell-Benjamin A.C. and Guinard J.X. (2003). Novel approaches and application of contemporary sensory evaluation practices in iron fortification programes. Critical Reviews in Food Sci. and Nutr. 43: 379 400.

Burch H.B. and Siegel A.L. (1971). Improved method for measurement of 6-a-levulinic acid dehydratase activity of human erythrocytes. Clin. Chem. 17 (10):103.

Dacie J.V. and Lewis S. M. (1975). Practical hematology. A collaborative analysis of an improved produce. 1 st. ed, John Wiley \& Sons, Inc: New York.

Dary O. and Mora J. O. (2002). Food fortification to reduce vitamin A deficiency: Intl. Vitamin A Consultative Group recommendations. J. Nutr. 132(Suppl 9):2927S-33S.

Davidsson L., Kastenmayer P. and Szajewska H. (2000). Iron bioavailability in infants from an infant cereal fortified with ferric pyrophosphate or ferrous fumarate. Am. J. Clin. Nutr. 71:1597-602.

Diaz-Castro J., Ojeda M. L., Alferez M. J. M., Lopez-Aliaga, I., Nestares T. and Campos M.S. (2011). Se bioavailability and glutathione peroxidase activity in iron deficient rats. J. Trace Elements in Medicine and Biology 25: 42-46.

Diosady L.L., Alberti J.O. and Venkatesh Mannar M.G. (2002). Microencapsulation for iodine stability in salt fortified with ferrous fumarate and potassium iodide. Food Res. Inter. 35: 635-642.

Eder K. and Kirchgessner M. (1996). Zinc deficiency and the desaturation of the linoleic acid in rats force fed fat free diets. Bio. Trace Element Res. 54: 173 - 183.

Fang X., Shima M. and Adachi S. (2005). Effects of drying conditions on the oxidation of linoleic acid encapsulated with gum arabic by spray drying. Food Sci. Tech. Res. 11:380-384.

Feigin R.D. (1977). Interaction of nutrition and infection: plans for future research. Am. J. Clin. Nutr. 30: 1553-1563.

Fossati P. and Prencipe L. (1982). The determination of triglycerdes using enzymatic methods. Clin. Chem. 28: 2077.

Fox T.E., Eagles J. and Fairweather-Tait S.J. (1998). Bioavailability of iron glycine as a 
fortificant in infant foods. Am. J. Clin. Nutr. 67: 664- 668 .

Gharsallaoui A., Roudaut G., Chambin O., Voilley A. and Saurel R. (2007). Applications of spray-drying inmicroencapsulation of food ingredients. Food Res. Int. 40 (9) : 11071121.

Gibson R. S. (2007). The role of diet- and hostrelated factors in nutrient bioavailability and thus in nutrient-based dietary requirement estimates. Food Nutr. Bulletin 28:77s-100s.

Gouin S. (2004). Microencapsulation: industrial appraisal of existing technologies and trends. Trends Food Sci. Techn. 15(7/8):330-347.

Hernandez M., Sousa V., Moreno A., Villapando S. and Lopez-Alarcon M. (2003). Iron bioavailability and utilization in rats are lower from lime-treated corn flour than from wheat flour when they are fortified with different sources of iron. J. Nutr. 133: 154-159.

Hernandez M., Sousa V., Moreno A., Villapando S., Lopez-Alarcon M. and Montalvo I. (2006). Cooking and $\mathrm{Fe}$ fortification have different effects on $\mathrm{Fe}$ bioavailability of bread and tortillas. J. the Amer. College of Nutr. 25(1): 20-25.

Hurrell R.F. (2002). Fortification: Overcoming Technical and Practical Barriers. J. Nutr.132: 806S.

Igarashi K., Nakanishi Y., Hirunuma R, Enomoto S. and Kimura S. (2006). Multi tracer study on the uptake of various trace elements in anemic rats: influence of NaFeEDTA and ferrous sulfate. Nutr. Res. 26:173- 179.

International Committee for Standardization in Hematology (1978). The measurement of total and unsaturated iron-binding capacity in serum. British J. hematology 38: 281.

Kelleher S.L. and Lonnerdal B. (2005). Low vitamin A intake affects milk iron level and iron transporters in rat mammary gland and liver. J. Nutr. 135: 27-32.

Kiskini. A., Kapsokefalou M., Yanniotis S. and Mandala I. (2012). Effect of iron fortification on physical and sensory quality of gluten-free bread. Food Bioprocess Tech. 5:385-390.

Knutson M. and Wessling-Resnick M. (2003). Iron metabolism in the reticuloendothelial system. Crit. Rev. Biochem. Mol. Biol. 38: $61-88$
Kuo T.M. and Gardner H.W. (2002). Palm stearin and oleic. Lipid biotechnology. $1^{\text {st }}$. ed., New York: CRC Press.

Kurtoglu E., Ugur A., Baltaci A.K. and Dar U.L. (2003). Effect of iron supplementation on oxidative stress and antioxidant status in iron-deficiency anemia. Biol. Trace Elem. Res. 96 (1-3):117-123.

Lee J.B., Ahu J., Lee J. and Kwak H. (2003). The microencapsulated ascorbic acid release in vitro and its effect on iron bioavailability. Arch. Pharm. Res. 26 (10): 874 - 879.

Leong W.I., Bowlus C.L., Tallkvist J. and Lonnerdal B. (2003). DMT1 and FPN1 expression during infancy: developmental regulation of iron absorption. Am. J. Physiol. Gastrointest Liver Physiol. 285:1153-1161.

Lim T.K. (2010). Plam stearin. Medicinal and non-medicinal edible plants. $1^{\text {st }}$., ed., Dordrecht:Springer. P.338.

Lin X.M., Wang Z., Shen X.Y., Long Z., Liu W.J., Guo Y.M. and Tang Y. (2003). Iron status and the effect of early iron supplementation on sub-clinical iron deficiency in rural school-age children from mountainous areas of Beijing (China). Zhonghua Yu Fang Yi Xue Za Zhi. 37 (2): 231-246.

Lobo A. R., Cocato M.L., Borelli P., Gaievski E.S.H., Crisma A.R., Nakajima K., Nakano E.Y. and Colli C. (2011). Iron bioavailability from ferric pyrophosphate in rats fed with fructan-containing yacon (Smallanthus sonchifolius) flour. Food Chem. 126: 885-891.

Martinez-Cervera S., Salvador A., Muguerza B., Moulay L. and Fiszman S.M. (2011). Cocoa fibre and its application as a fat replacer in chocolate muffins. LWT - Food Sci. and Tech. 44: 729-736

Martinez-Cervera S., Sanz T., Salvador A. and Fiszman S.M. (2012). Rheological, textural and sensorial properties of low-sucrose muffins reformulated with sucralose/ polydextrose. LWT - Food Sci. and Tech. 45: 213-220.

Parker M.M., Humoller F.L. and Mahler D.J. (1967). Determination of copper, zinc and other trace elements in biological material. Clin. Chem.13: 40.

Premkumar K. and Bowlus C.L. (2003). Ascorbic acid reduces the frequency of iron induced micronuclei in bone marrow cells of mice. 
Mutation Res. 542: 99-103.

Reeves P. G., DeMars L.C.S., Johnson W.T. and Lukaski H. C. (2005). Dietary copper deficiency reduces iron absorption and duodenal enterocyte hephaestin protein in male and female rats. J. Nutr. 135: 92-98.

Roodenburg J. C., West C. E., Howvenier R. and Beynen A.C. (1995). Evaluation of a twogeneration rat model for Vitamine A deficiency and the interrelationship with iron metabolism. British J. Nutr. 74: $689-700$.

Salgueiro J., Lysionek A., Ridolfi A., Zubillaga M., Barrado A., Martinez-Sarrasague M., Goldman C. and Boccio J. (2005). Nutritional and technological behavior of stabilized iron-gluconate in wheat flour. Bio. Trace Element Res.105:187- 195.

Soliman G.Z.A., Mahfouz M. H. and Emara I. A. (2010). Effect of different types of oral iron therapy used for the treatment of iron deficiency anemia and their effects on some hormones and minerals in anemic rats. J. American Sci. 6(6):109-118.

Strube Y. N. J., Beard J. L. and Ross A. C. (2002). Iron deficiency and marginal vitamin A deficiency affect growth, hematological indices and the regulation of iron metabolism genes in rats. J. Nutr. 132: 3607-3615.

Urbano G., Porres J. M., Frejnagel S., LopezJurado M., Gomez-Villalva E., VidalValverde C. and Aranda P. (2007). Improvement of iron availability from phytase-treated Pisum sativum, L. flour. Food Chemistry 103: 389-395.

Vulpe C.D., Kuo Y.M., Murphy T.L., Cowley 1., Askwith C., Libina N., Gitschier J. and Anderson G.J. (1999). Hephaestin, a ceruloplasmin homologue implicated in intestinal iron transport, is defective in the mouse. Nat. Genet. 21: $195-199$.

Wallach J. (1992). Interpretation of Diagnostic Tests. A Synopsis of Laboratory Medicine, $5^{\text {th }} \mathrm{Ed}$. A little brown handbook, Boston.

Wastson D.A. (1960). Simple method for the determination of serum cholesterol. Clin Chem Acta 5:589.

Wegmuller R., Zimmermann M.B. and Hurrell R.F. (2003). Dual fortification of salt with iodine and encapsulated iron compounds: stability and acceptability Testing in Morocco and Cote d'Ivoire. J. Food Sci. 68 (6):2129 - 2135.

Wieland H. and Seidel D. (1983). A fully enzymatic colorimetric determination of HDL-cholesterol in the serum. J. Nutr. 109: $760-766$.

Wienk K. J., Marx J. J. and Beynen A.C. (1999). The concept of iron bioavailability and its assessment. European J. of Nutr. 38:51-75.

Windisch W. (2002). Interaction of chemical species with biological regulation of the metabolism of essential trace elements. Analytical and Bioanalytical. Chem. 372: 421-425.

Wintrobe, M.M. (1967). The Wintrobe Erythrocytic Indexes. Clinical Haematology. $6^{\text {th }}$ _ed., Lea \& Febiger, Phila. P: $414-419$.

Yao O. L., Diosady L.L. and Wesley A. S. (2010). Iodine stability in iodized salt dual fortified with microencapsulated ferrous fumarate made by an extrusion-based encapsulation process. J. Food Engineering 99: 232-238.

Yeung C. K., Miller D.D., Cheng Z. and Glahn R.P. (2005). Bioavailability of elemental iron powders in bread assessed with an in vitro digestion/Caco-2 Cell Culture Model. J. Food Sci. 70: (3): 199S- 203S.

$$
\begin{aligned}
& \text { التقييم البيولوجى للكيك (الموفينية ) المدعم بالحديد فى الصوره الكبسوليه } \\
& \text { داليا محم النحال } \\
& \text { قسم الأغذيه الخاصده و التغذيهـ معهد بحوث تكنولوجيا الأغذيهـ مركز البحوث الزر اعيهـ الجيزه ـ مصر }
\end{aligned}
$$

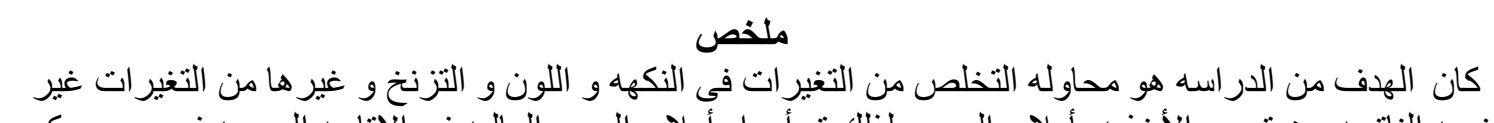

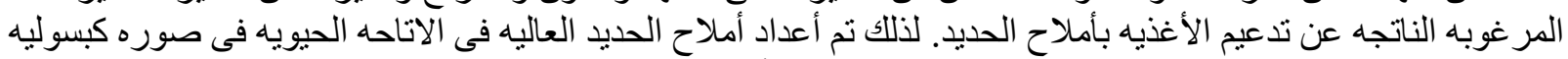

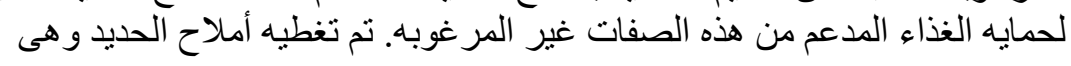

(ferrous fumarate, Fe Na EDTA, ferrous glouconate, ferrous citrate, ferrous carbonates and ferrous sulphate anhydrous) 
بأستيارين النخيل فى وجود الليسيسين و حمض الأسكوربيك. و حتى يتم إختبار أملاح الحديد المكبسله فقد تم تدعيم الدقيق الداقي

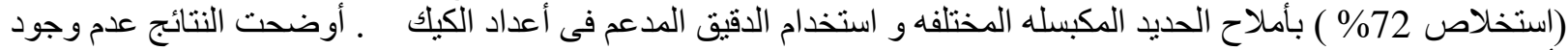

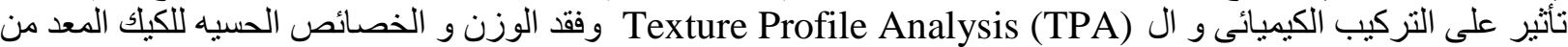

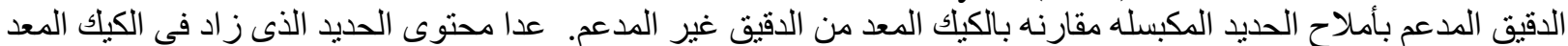

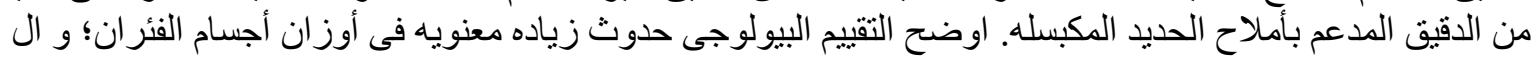
hematological apparent digestibility coefficient (ADC) parameters

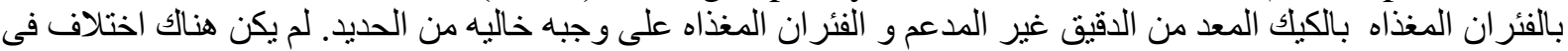

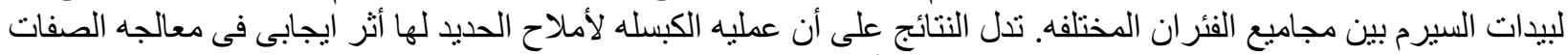

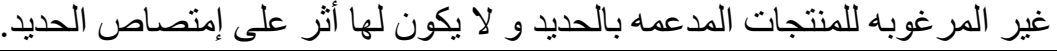

المجلة العلمية لكلية الزراعة - جامعة القاهرة - المجلد (63) العدد الثالث (يوليو 2012): 286- 298 ـ 